\title{
STATUS OF THE EUROPEAN SPALLATION SOURCE DESIGN STUDY
}

\author{
I S K Gardner ${ }^{1}$, H Lengeler ${ }^{2}$, K Bongardt ${ }^{2}$, H Klein ${ }^{3}, \mathrm{G} \mathrm{H}$ Rees ${ }^{1}$ and C M Warsop ${ }^{1}$ \\ on behalf of the ESS Study Group. ${ }^{1}$ Rutherford Appleton Laboratory (RAL), UK; \\ ${ }^{2}$ Forschungszentrum Julich (FZJ), Germany; ${ }^{3} \mathrm{~J}$ W Goethe Universitat (JWGU), Germany.
}

\section{Abstract}

The status of a study for a European Spallation Source (ESS) is reported along with a description of the reference design. The design of the $5 \mathrm{MW}, 1.334 \mathrm{GeV}$ machine is dominated by the need to keep beam loss at a low enough level to allow hands on maintenance for most areas. All accelerator sections are designed to be far away from space charge limits. The design allows scraping of the $\mathrm{H}^{-}$Linac beam in all three phase planes (horizontal, vertical and momentum) prior to injection into the two accumulators. Key elements of the R \& D programme that will validate the design are identified.

\section{ESS DESIGN STUDY STATUS}

The first phase of the ESS Project was completed at the end of 1996, with the conclusion of the Feasibility Study and publication of the Reference Design [1]. This work identified areas of research required to validate the design, and is the basis for planning the next ' $R \& D$ ' Phase of the study which is outlined in Section 6.

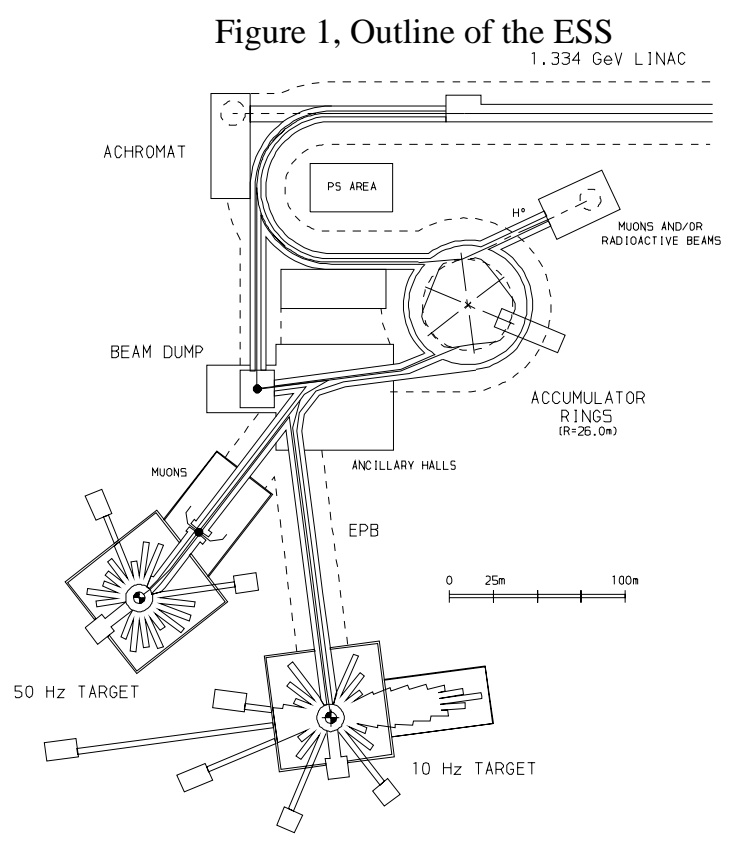

\section{ESS REFERENCE DESIGN}

The Reference Design consists of a $1.334 \mathrm{GeV} \mathrm{H}^{-}$ Linac, which develops the full $5 \mathrm{MW}$ beam power at $50 \mathrm{~Hz}$, followed by two Accumulator Rings which operate in parallel to compress the proton pulse to $1 \mu \mathrm{s}$.
Two spallation targets are included, one for $50 \mathrm{~Hz}$ operation and the other for $10 \mathrm{~Hz}$, see Figure 1.

The Linac provides a peak current of $107 \mathrm{~mA}$ (at 6\% duty cycle) over two successive $0.6 \mathrm{~ms}$ injection pulses into each ring. To minimise injection losses, the $1.2 \mathrm{~ms}$ injection pulse is chopped at the ring revolution frequency of $1.67 \mathrm{MHz}$, and linac halo is removed in a long collimation section in the injection line. Charge exchange injection is over 1000 turns, accumulating $2.34 \times 10^{14}$ protons per ring. Fast extraction from each ring, on successive turns, yields the $1 \mu$ s pulse required.

\section{THE ESS LINAC}

Two $70 \mathrm{~mA} \mathrm{H}^{-}$ion sources feed RFQs whose beams are funnelled together at $5 \mathrm{MeV}$ into a $350 \mathrm{MHz}$, $70 \mathrm{MeV}$ DTL. Next, a $700 \mathrm{MHz}$ coupled cavity linac accelerates the beam up to the final energy of $1.334 \mathrm{GeV}$. See Figure 2. The momentum of the beam is ramped and the spread is reduced by a bunch rotator. Hands on maintenance is a more important criterion than either capital or operating cost minimisation.

\section{Figure 2, Proposed Linac Scheme}

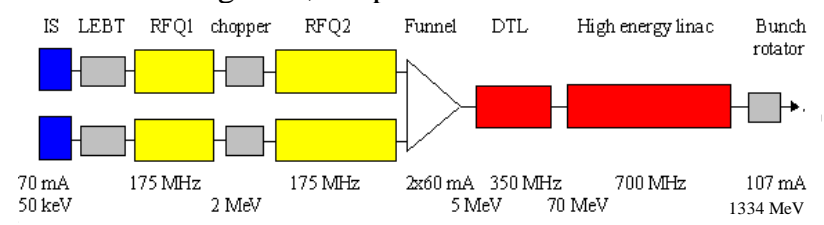

\subsection{Low Energy Section}

The $\mathrm{H}^{-}$ion source requirements of $70 \mathrm{~mA}$ at $6 \%$ duty cycle have not yet been achieved. The most promising candidates are developments of the ISIS Penning source, which has already delivered $55 \mathrm{~mA}$, or an RF driven volume source [1]. As neutralisation effects in the transport system between the ion source and RFQ have not been demonstrated for $\mathrm{H}^{-}$at high intensities, an integrated ion source-RFQ ESS test stand is to be built at RAL. Various parts will be delivered by the ESS collaborators. Details of the $2 \mathrm{MeV}$ chopping line and the $5 \mathrm{MeV}$ funnelling line are given in [1].

\subsection{DTL and CCL Linacs}

After acceleration to $70 \mathrm{MeV}$ in a conventional $350 \mathrm{MHz}$ DTL, the particles are injected into a $700 \mathrm{MHz}$ CCL. The injection energy is below the neutron production threshold of $\sim 120 \mathrm{MeV}$. The CCL at high 
energy is the most sensitive for beam loss. It is also the most expensive, for both capital and operating cost.

Optimisation of capital and 10 years operating costs leads to a field gradient of $2.8 \mathrm{MV} / \mathrm{m}$ and length $663 \mathrm{~m}$. By decreasing the full current transverse tune with energy, even for the $214 \mathrm{~mA}$ bunch current at $700 \mathrm{MHz}$, the transverse and longitudinal tune ratios are $\sim 0.8$ all along the ESS linac. This non space charge dominated design leads to rms emittance growth of $<10 \%$. Most important for beam loss is the reduced halo outside the dense core. Monte Carlo simulations with $\leq 10^{6}$ particles have been run for the DTL and the CCL in sequence [2]. There is strong transverse-longitudinal coupling associated with the non-uniform space charge density of a bunched beam. The linac is expected to achieve the low loss design goal after an initial start-up period.

\subsection{RF Power for CCL}

Each klystron feeds only two cavities and is designed to deliver $2 \mathrm{MW}$ peak RF power, which is $30 \%$ more than the sum of beam and cavity dissipation power. No modulating anode is foreseen for this single beam klystron. A bouncer has been designed for a modulator delivering $6 \mathrm{MW}$ DC power for $1.2 \mathrm{msec}$ pulses at $50 \mathrm{~Hz}$. The AC to DC efficiency is about $85 \%$ and the pulse flatness is better than $\pm 0.5 \%$ [1]. This modulator may be used to power a pair of $2 \mathrm{MW}$ peak RF power klystrons. The replacement of Gate Turn Off (GTO) thyristor switches by Insulated Gate Bipolar Transistors (IGBT) is promising for reducing costs and control problems. A test stand is planned for studying the series connection of IGTBs up to a blocking voltage of $5 \mathrm{kV}$ for each. Costs are reduced as all modulators are designed for the same DC power; a central housing of modulators for many klystrons using common energy storage and control systems is possible. More detailed studies are planned to evaluate this arrangement.

Attention has to be given to the RF control system. Uncorrelated amplitude and phase errors, of $\pm 1 \%$ and $\pm 1^{\circ}$ respectivley, will cause an oscillation of the beam centre of about $0.6 \mathrm{MeV}$ at the linac end. This is near the limit for low loss ring injection.

\subsection{Superconducting Option}

Superconducting cells are an interesting option for the high $\beta$ linac. Detailed studies are to be performed for accelerating the ESS beam from $70 \mathrm{MeV}$ to $1.334 \mathrm{GeV}$ by using 5-cell $700 \mathrm{MHz}$ superconducting $\mathrm{Nb}$ cavities at $2 \mathrm{~K}$. A constant gradient of $10 \mathrm{MV} / \mathrm{m}$ is chosen. Two cavities are connected to one klystron. The total linac length is $300 \mathrm{~m}$. About $30 \%$ saving of capital and operating cost is expected for this sc linac compared to the nc reference design, if amplitude and phase can be stabilised to better than $\pm 1 \%, \pm 1^{\circ}$ respectively. RF errors due to the Lorentz force detuning may be compensated by stiffening the cavities and applying digital feedback from feedforward tables. The cavity to cavity and pulse to pulse frequency shift of $\pm 30 \mathrm{~Hz}$, as measured for a stiffened $1.3 \mathrm{GHz}$ sc TESLA cavity due to microphonics, causes an intolerable phase shift of $\pm 3^{\circ}$ at low $\beta$ and $\pm 1.2^{\circ}$ at high $\beta$. Unlike electron linacs, phase errors in different cavities connected to one klystron do not add up to give a common energy fluctuation. To achieve the very stringent limits required, methods such as connecting only one cavity to a klystron, or increasing the cavity bandwidth by lowering the loaded Q-value, are being considered in cooperation with the TESLA RF control group at DESY, Hamburg.

\section{THE INJECTION TRANSFER LINE}

The injection line is involved with collimation, momentum ramping and bunch rotation. For low loss in the rings, linac halo is removed. In the transverse planes unnormalised emittances are collimated at $2.54 \pi \mathrm{mm} \mathrm{mr}$. To allow phase space painting in the ring, the momentum is ramped linearly through injection. The instantaneous $\delta p / p$ is adjusted with bunch rotator cavities, which compensate for space charge induced phase width growth. The total momentum range, spread plus ramp, is collimated at $0.0 \%>\delta \mathrm{p} / \mathrm{p}>0.4 \%$.

There are three sections; the initial drift and matching section, an achromatic bending region, and a final vertical separation and optical matching section. All collimation systems in the line utilise stripping foils and magnet fields to separate and remove unwanted beam efficiently. Magnets have $\mathrm{C}$ shaped cores, open on the outer radius for easy removal of stripped beam.

The first section includes the momentum ramping cavity and debuncher at the upstream end, four horizontal betatron collimators at successive phases of $45^{\circ}$ and, at the down stream end the bunch rotator. The $180^{\circ}$ achromatic bending region has a mean radius of $42.5 \mathrm{~m}$ to keep fields low and prevent $\mathrm{H}^{-}$stripping. This provides a peak normalised dispersion of $5.5 \mathrm{~m}^{1 / 2}$ for momentum collimation; two momentum pre-collimators allow reduction of magnet apertures required. The achromat has four superperiods, each containing low field combined function magnets in a triplet configuration. Beam dynamics are similar to a circular machine above transition, with minimal debunching. Four vertical betatron collimators are also included in the achromat, placed at relative phases of $45^{\circ}$. After the achromat the line splits into two ring matching sections, $2 \mathrm{~m}$ vertically apart. A vertical splitting magnet, with a rise time of $100 \mu \mathrm{s}$, deflects the beam appropriately.

\section{ESS ACCUMULATOR RINGS}

\subsection{Accumulator Lattice and Injection}

The $1.334 \mathrm{GeV}$ accumulators have a mean radius of $26 \mathrm{~m}$, and a three superperiod lattice utilising quadrupole 
triplets to provide alternate achromatic bending regions and long dispersionless straights. This fullfills requirements for extracted pulse length, low loss injection, RF, extraction and collimation. Beam emittances are large (collimated at $260 \pi \mathrm{mm} \mathrm{mr}$ ) to keep betatron tune depressions below 0.1 at the design intensity of $2.34 \times 10^{14}$ per ring. For full design see [1].

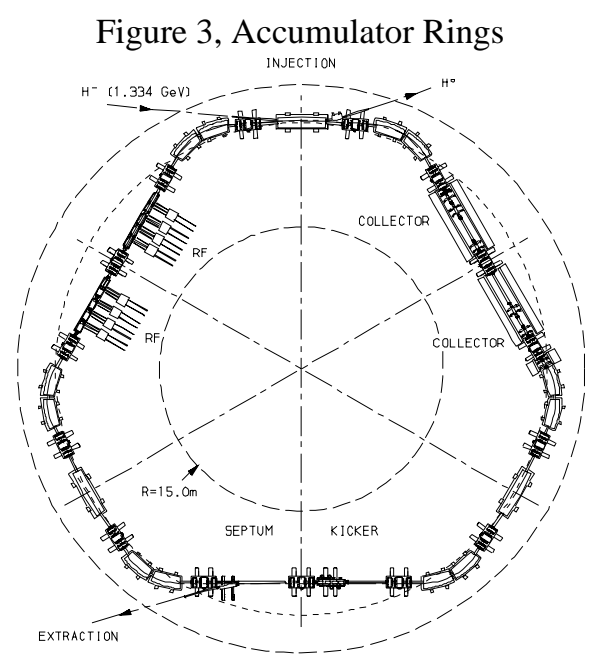

During injection the beam is painted in all three planes to minimise space charge effects. The beam momentum is ramped linearly through injection and the large dispersion at the foil $(5.66 \mathrm{~m})$ correlates this longitudinal painting to that in the horizontal plane. Vertically a programmed symmetrical bump at the foil anti-correlates vertical amplitudes. One topic for research is the effect of space charge on the painted transverse emittance and related loss.

The injection system requires careful design, main requirements are: specified dispersion for injection painting; efficient removal and collection of $\mathrm{H}^{\circ}, \mathrm{H}^{-}$; low field to prevent pre-stripping and efficient collection of stripped electrons to prevent foil heating. Manufacture and verification of graphite foil performance is part of the $R \& D$ program. Interactions of recirculating protons with the foil cause losses, and measures to minimise the number of foil traversals are: collapsing vertical orbit bump, optical mismatch at injection, RF steering after injection, two unsupported edges on the foil and the large machine acceptance.

\subsection{RF System and Extraction}

The chopped linac beam is injected unmatched into the bucket of the dual harmonic RF system $(h=1,2)$. RF voltage and frequency laws have been determined by stability and injection criteria, and optimised with space charge codes [3]. There are four RF cavities per ring, providing a peak total voltage of $27 \mathrm{kV} /$ turn. A small frequency sweep is used for final RF steering, and a $200 \mathrm{~ns}$ beam gap allows for the rise time of the single turn extraction system. Extraction uses four push-pull lumped kickers, which deflect the beam by $15.8 \mathrm{mr}$ to a septum magnet half a straight downstream.

Precautions against instabilities include profiled aluminium vacuum chambers, shielded bellows, low impedance extraction kickers and further R\&D work.

\subsection{Loss Collection in Accumulator Rings}

Total loss in each ring is estimated at $<0.02 \%$ and is mainly due to foil interactions. Most loss will be localised in a well shielded, dispersionless collection region. Collimation and aperture limits are 260 and $480 \pi \mathrm{mm} \mathrm{mr}$ respectively. Most operational loss will be stopped on a betatron collimation system and a special momentum tail system. The latter intercepts particles losing energy at the high dispersion point of the foil, by intercepting their enhanced betatron motion at $180^{\circ}$ phase shift downstream. The system of collimators is being optimised for most efficient collection of particles, including effects of growth rate, outscattering, misalignment and fault conditions.

\section{RESEARCH \& DEVELOPMENT PROGRAM}

An application for CEC support under the TMR scheme has been submitted. Research topics and participants are summarised in Table 1 for the 3 year TMR R\&D program. An ESS test stand with ion source, chopper and RFQ is to be built at RAL, and beam loss studies will continue.

Table 1, Summary of Topics and Participants

\begin{tabular}{|l|l|}
\hline Subject & Participants \\
\hline Ion Sources & RAL, JWGU \\
\hline 2 MeV RFQ & RAL, JWGU, TUE, FZJ, HMI \\
\hline Chopper Design & TSL, JWGU, TUE, FZJ, HMI \\
\hline Space Charge Codes & FZJ, RAL JWGU, INFN \\
\hline Foil \& Injection Studies & RAL, HMI \\
\hline Diagnostics & U Aarhus, TSL, FZJ, HMI, JWGU \\
\hline Linac Cavity and RF & TUE, FZJ \\
\hline Impedance Modelling & INFN, RAL \\
\hline
\end{tabular}

Participants are: RAL, JWGU, FZJ, Univ. of Aarhus (U. Aarhus), The Svedberg Laboratory (TSL), Istituto Nazionale di Fisica Nuclear (INFN), Eindhoven Univ. of Technology (TUE), Hahn Meitner Institut (HMI). Other institutes may join as studies progress.

The anticipated ESS programme is: completion of R\&D by 2000, two years engineering design, six years construction, two years commissioning ending by 2010.

\section{REFERENCES}

[1] ESS A Next Generation Neutron Source for Europe, Volume 3, The ESS Feasibility Study, March 1997.

[2] M Pabst et al, Proc. of this conference.

[3] C Prior, ESS Report, ESS 94-7-R, November 1994. 